\title{
Blockchain Technology : A Tool to Secure Data Namrata Thakur ${ }^{1}$, Dr. Vinayak D Shinde ${ }^{2}$
}

${ }^{1}$ Department of Computer Engineering, Shree L.R.Tiwari college of Engineering, Mira Road (E), Thane, Maharashtra, India

${ }^{2}$ Associate Professor, Department of Computer Engineering, Shree L.R.Tiwari college of Engineering, Mira Road (E), Thane, Maharashtra, India

Article Info

Volume 7, Issue 4

Page Number: 434-438

Publication Issue :

July-August-2021

\section{Article History}

Accepted : 20 July 2021

Published : 30 July 2021

\section{ABSTRACT}

Data security is the key to the development of modern Internet technology. The distributed, decentralized, and secured hashed mechanism of the blockchain gives a complete new point of view for the evolution of data security technology. Block chain technology is one of the major technological innovations of this century. In the last couple of years, the interest around blockchain technologies is increasing. Many implementation of blockchain technology are widely available today. Blockchain,the foundation of Bitcoin, has gain much attention in this era. Blockchain is an encrypted, immutable, distributed ledger, which allows transactions take place in a decentralized manner. Blockchain based applications expected to alter numerous fields including financial services, health care, entertainment media, Internet of Things (IoT), and many more. The Blockchain technology plays important role in the process of data security. In this paper, we will discuss about the research being done on this new domain of Computer Science. It is not only the most popular topic to discuss about, but is the most technological innovation, that is all set to reform the entire world.

Keywords : Blockchain Technology, Security, DLT, Blocks.

\section{INTRODUCTION}

As technology continues to be a constantly evolving field,a number of developments have shaped its course.Block chain technology is now the center of discussion as lot of industries are moving towards implementing this new development[1].Nowadays, new technologies are being adopted within many sectors, such as public, industrial, energy as well as the service sector. Many pioneer works done to improve the efficiency of Blockchain. According to the research done by Zhao et al. (2016), we have found that there has been a large-scale increase in the number of research papers on Blockchains. Blockchains has considered as exposed to many of the attacks.

Blockchain a Distributed Ledger Technology (DLT) is a revolutionary technology that enables transacting data in a decentralized structure without trusted central authorities [6]. People do not trust one another with their personal data, so they need to share their data in a secured and tamperproof platform and blockchain helps in creating that 
platform. Looked upon as one of the most breakthrough technologies across industries, which results in, reduce costs, increase transparency, enhance security and increase efficiency among others.

\section{BACKGROUND AND RELATED WORK}

Computer security always revolves around data, device \& network security. Data builds the foundation of the application system, and its integrity is the key to the data's value and the goal of data security technology protection. Now a day's data is becoming one of the most valuable resources in the world. Data security provides set of standards and technologies that protect data from intentional or accidental destruction, modification or disclosure. The main objective of data security is to protect the organization data. Encryption, masking, erasure, resilience, administrative controls, physical security, logical controls, organizational standards, and other safeguarding techniques used to apply security to data. Compliance is also a major consideration. It doesn't matter which device, technology or process is used to manage, store or collect data, Data must be protected irrespective of device, technology or process used to manage, store or collect data. Data breaches can result in legal action cases and huge fines against organization reputation.

Nowadays Blockchain as an Alternative technology plays a significant role in securing data transparency, change escalation, and fine-grained accessibility of data information, which is significant for data operating organizations that deal with large amounts of sensitive data and suffer from frequent hacker attacks. Everything is encrypted and nobody can tamper with the blockchain without everyone else noticing immediately.

As shown in Figure-1 block chain is also similar to a database which stores information however the main difference is that the data is located in a network of personal computers called nodes where there is no central entity such as government or bank controlling
data.A Blockchain is basically a decentralized, distributed ledger of all the transactions or events which takes place only after involving multiple parties. It ensures high level of security as the transactions, which take place, are entirely anonymous. Each transactions or digital events taking place in a Blockchain network is verify only if it is agree upon by the consensus of the majority party of the users participating in this process. The interest in Blockchain technology has been increasing since the idea coined in 2008. The reason for the interest in Blockchain is its central attributes that provide security, anonymity and data integrity without any third party organization in control of the transactions, and therefore it creates interesting research areas.
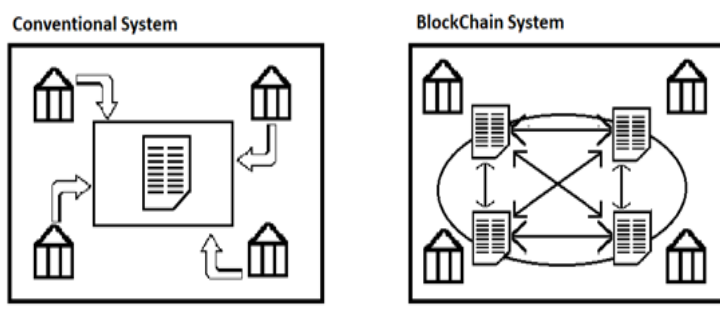

Figure-1 :- Distributed ledger \& Blockchain Technology

\section{Overview of Blockchain Technology}

Blockchain is a decentralized database, which keeps record of every transaction done on a network. Rather than having a traditional central database like that of banks or governments, it has a ledger distributed over a network of nodes [1]. This network can be public, like the internet, which is accessible to any person in the world or it can be private, with accessibility given to only members of an organization. Blockchains technology allows users to trust each other and make peer-to-peer transactions, without third party. This technology is not only affecting the way we use internet, but the global economy is also being revolutionized [7]. 
Namrata Thakur et al Int. J. Sci. Res. Comput. Sci. Eng. Inf. Technol, July-August-2021, 7 (4) : 434-438

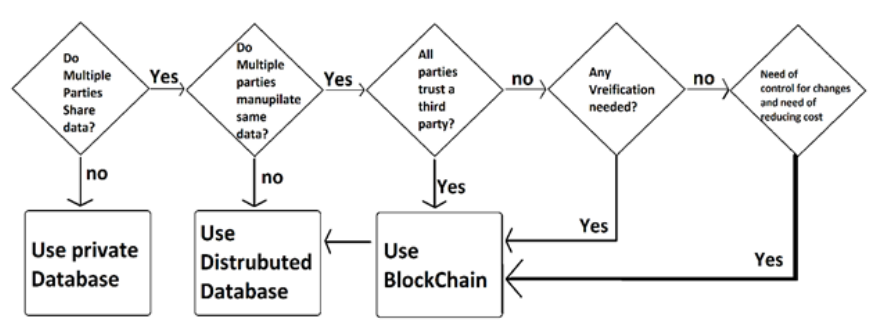

Figure 2 :- Use of Blockchain Technology

\section{Blockchain Architecture}

Blockchain is a sequence of blocks, which holds a complete list of transaction records like conventional public ledger. Figure 3 shows an example of a blockchain architecture [2]. As shown in Figure 3 a blockchain is the linked list of blocks. Each block refers to its previous block just like page number in book. It consists of only one parent block called as Genesis block and block header contains previous block hash value. Genesis blocks have no parent block. [2]Other blocks in chains called as children blocks. Hash values of all blocks would be stored in ethereum block chain [6].

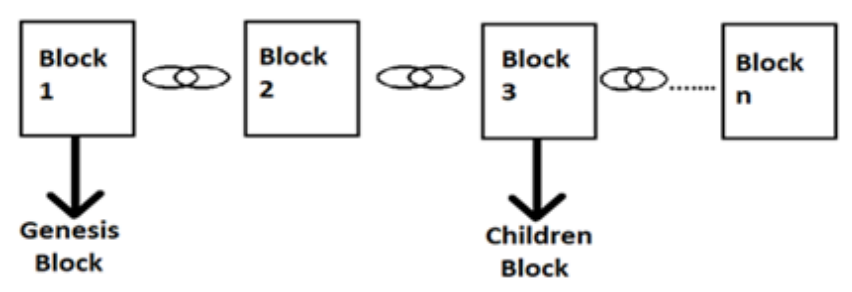

Figure 3 :- Blockchain Architecture

\section{- Block}

A block in a chain has block header and the block body as illustrates in Figure 4 [6]. In addition, the block header contains-
a. Block version: indicates which set of block validation rules to follow.
b. Merkle tree root hash: the hash value of all the transactions in the block.
c. Timestamp: current time as seconds in universal time since January 1, 1970.
d. nBits: target threshold of a valid block hash.

e. Nonce: an 4-byte field used for hash calculation

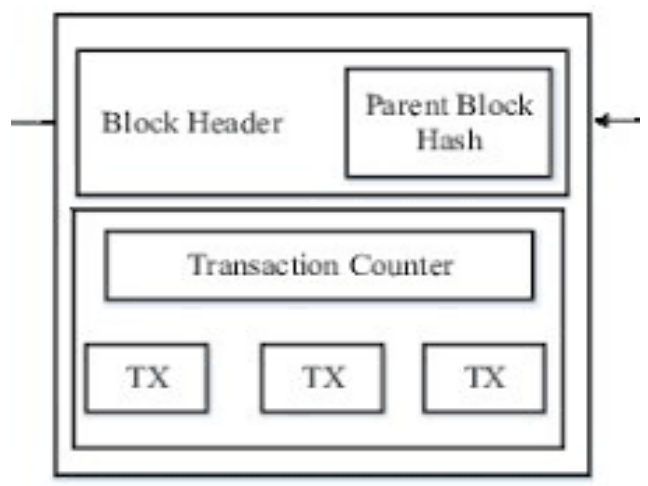

\section{Block i}

Figure 4 :- Block structure

Parent block hash: a 256-bit hash values those points to the previous block.Along with this the body of block has transaction counter and transactions. The maximum number of transactions of blocks depends on the block size and the size of each transaction [6].

\section{Blockchain for Data Storage}

Blockchain can be use in distributed storage software by different ways. One of the most common way is by breaking data into different chunks followed by encryption and Distribute files across the whole network so that all your files are available in case of network is slow.

\section{Blockchain implementation done in three domains:-}

1. Public blockchain:- In this information can read and send by any participant. Examples are Etherum and Hyperledger project.

2. Private blockchain belongs to one institute. Example is Microsoft.

3. Consortium blockchain, a set of preselected participants are control the consensus process.

\section{Benefits of blockchain technology}

1. Users control all their information and transactions. 
2. Blockchain data is complete, consistent, accurate, and universally available.

3. Blockchain network does not have any center point of failure since its decentralized results in reduced risk.

4. Users can trust transactions will executed exactly as the protocol commands removing the need for a trusted third party [4].

5. Changes made in public blockchains are viewable by all parties creating transparency, and all transactions are immutable, meaning they could not be altered or deleted.

6. Blockchain transaction can reduce transaction times to minutes and are processed 24/7.

7. The transactions that take place are transparent. The individuals provided with authority can view the transaction.

\section{Possible Problems and Limitations}

1. Security is limited by the size of the network .it becomes vulnerable to attacks if size of network is larger [4].

2. In case of small and or unreliable network, more redundancy may needed than other storage models [4].

3. Network communication overhead can be huge and suffers from interoperability.

4. Network bandwidth could be a problem as seen with Bitcoin.

\section{Result and Implementation}

A blockchain represents the linked list of blocks. A blockchain is immutable, which means once the block added to the blockchain it could not be alter. Basic models of the Blockchain created in JavaScript using Node Js.Figure 5 shows how blocks in the block chains are created and how mining done and credit given to mining is shown in figure 6 and figure7 respectively[3].

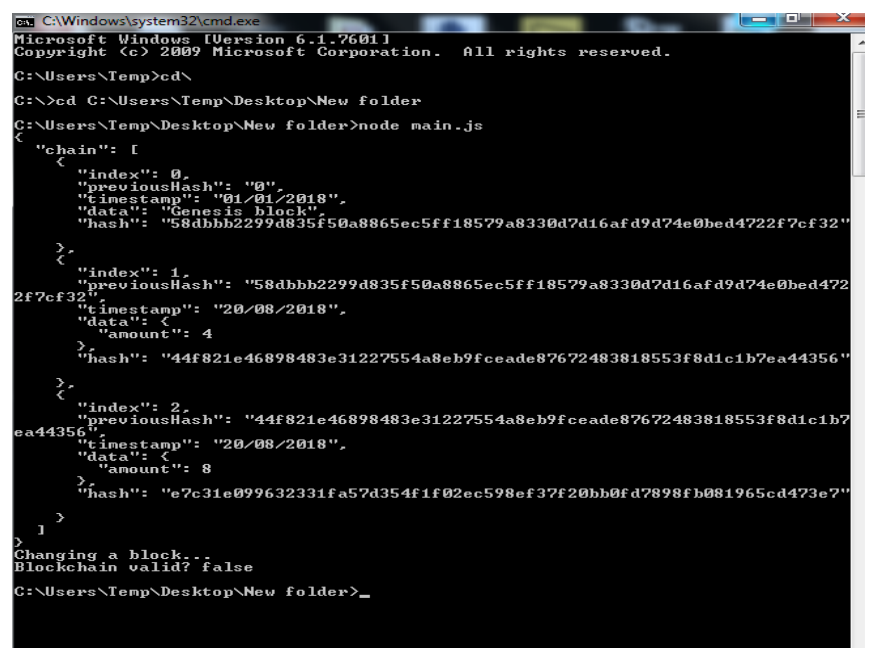

Figure 5:- Creation of Blocks

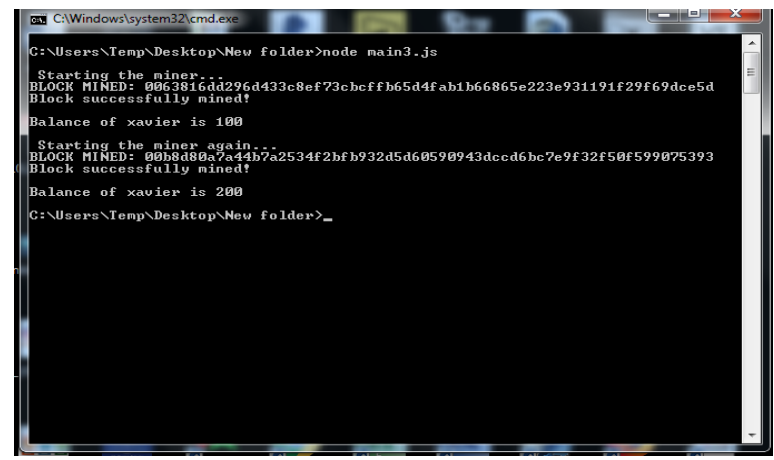

Figure 6:- Block mining

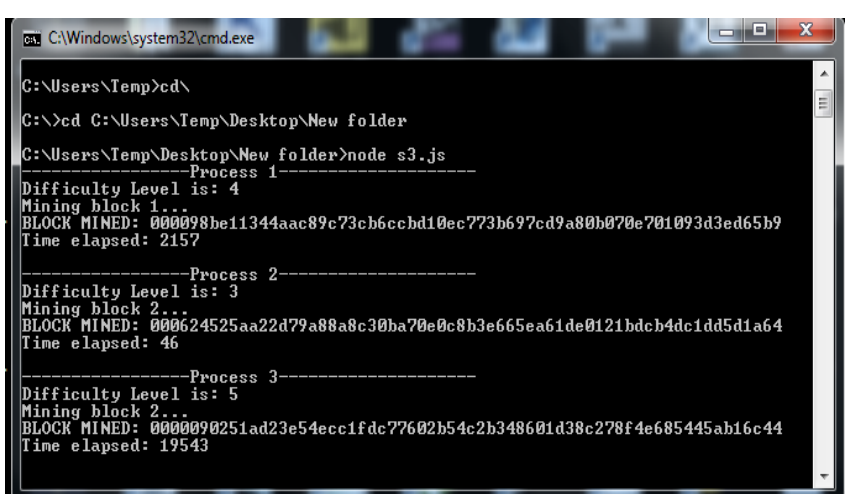

Figure 7:- Comparison of time taken for mining

\section{Future Work}

Blockchain applications developed daily and can considered as the most effective tools of the decade. Our society yet not to be aware of the brilliance of this technology, since this lack of understanding results in less ultimate success. The best way to recognize its potential is to analyses block 
chain applications examples \& after the overall observation of this I want to do the further research \& implementation on blockchain.

\section{CONCLUSION}

By the end of 2024 as per the reports by Transparency Market Research, the global blockchain technology market is projected to be worth $\$ 20$ billion. [1]The researchers in the domain of security and Cryptography have come forward to take it further to newer highs. Blockchain technology is going to provide a great help for the financial and NonFinancial sectors. Blockchain technology is a new way based on key technologies such as password security, decentralized, shared public accounts and provides reliability, security and shared knowledge visibility of its proper controls and permissions. However, blockchain technology is expected to bring a revolutionary solution to the unmanaged database. This paper could conclude that there are numerous opportunities of research in this area and there is an urgent need to explore and seek for betterment just by minimizing the flaws and by enhancing its efficiency.

\section{REFERENCES}

[1]. Rishav Chatterjee ,Rajdeep Chatterjee, “An Overview of the emerging technology:Blockchain", International Conference on computational intelligence and network ,2017, Odisha ,India

[2]. Sachidanand Singh, Nirmala Singh ,"Blockchain: Future of financial and cyber security", 2016,International conference on contemporary computing \& informatics, DOI 10.1109/IC3I.2016.7918009

[3]. Massimo Di Pierro, "What is the blockchain?", 2017,IEEE,DOI 10.1109/MCSE.2017.3421554 [4]. Pinyaphat Tasatanattakool, Chian
Techapanupreeda, "Blockchain :Challenges \&
Application",2018,IEEE,

DOI 10.1109/ICOIN.2018.8343163

[5]. Antony Louis," A Gentle Introduction to blockchain Technology".2018,BNC ,p.p1-18

[6]. Zibin Zheng1, Shaoan Xie1, Hongning Dai2, Xiangping Chen4, and Huaimin Wang3,"An Overview of Blockchain Technology:Architecture, Consensus, and Future Trends",2017,IEEE 6th International Congress on Big Data,DOI 978-1-53 86-1996$4 / 17$

[7]. MorgenPeck,"Blockchain World", 2017,IEEE Future Directions Blockchain Initiative, White paper Blockchain incubator.

\section{Cite this article as :}

Namrata Thakur, Dr. Vinayak D Shinde, "Blockchain Technology : A Tool to Secure Data", International Journal of Scientific Research in Computer Science, Engineering and Information Technology (IJSRCSEIT), ISSN : 2456-3307, Volume 7 Issue 4, pp. 434-438, July-August 2021. Available at doi : https://doi.org/10.32628/CSEIT2174111 Journal URL : https://ijsrcseit.com/CSEIT2174111 